and noninfectious to humans. The ability of the virus to penetrate the fabric is assessed by a culturing technique.

Manufacturers of protective materials have praised the development of these standards because, until now, there has been no universally acceptable test method. Critics of the test method charge that the tests do not necessarily simulate the "conditions of use," that is, the amount of pressure and time the materials are exposed to the liquid. Concern has been raised by some users regarding the clinical implications, that is, whether all protective barriers, regardless of the amount and type of anticipated exposure to blood and body fluids, would be required to pass these ASTM tests.

These test methods were developed by the ASTM Committee F23 on Protective Clothing and issued as emergency standards by ASTM for a period of only two years. Comments will be taken from the user community throughout this two-year period and will be used to evaluate the standards and the need to change their status to permanent, full-consensus standards.

Norman W. Henry, chairman of a subcommittee of F23 has said that "despite skepticism about the test conditions and methods, at least we have a consensus. These are emergency standards and can always be improved."

ASTM invites interested parties to participate in the development of these and other standards within Committee F-23. The next committee meeting is scheduled for June 23-25, 1993, in Atlanta. For more information, contact Norman W. Henry III, E.I. du Pont de Nemours \& Co., Haskell Laboratory, Elkton Rd., PO. Box 50, Newark, DE 19714. Telephone (302) 366-5250.

\section{Loofah Sponges Added to List of Source for Pseudomonas aeruginosa Pustular Folliculitis}

Whirlpools, swimming pools, and hot tubs have been reported as sources of Pseudomonas aeruginosa causing cutaneous infections. Such infections are characterized by small pustular lesions on an erythematous base involving the skin of the trunk, buttocks, and extremities. A recent report indicates another source for this infection.

A 25year-old woman was reported to have a two-week history of perifollicular pustular lesions on her abdomen, thorax, and lower extremities. The lesions first appeared on her abdomen and then progressed to her legs. She then developed tender axillary lymphadenopathy. Gram stain of several pus- tules revealed many polymorphonuclear leukocytes and gram-negative bacilli and Pseudomonas aeruginosa was recovered from the culture.

The patient denied using spas, hot tubs, or swimming pools but reported using a loofah sponge that was kept hanging in her shower. Cultures of the sponge yielded heavy growth of $\mathbf{P}$ aeruginosa that was identical by serotyping and pyocin typing to the skin isolate. Although newly purchased loofah sponges yielded only a few colonies of Staphylococcus epidermidis and Bacillus species, sterilized sponges served as an excellent culture medium when inoculated with $\mathbf{P}$ aeruginosa $(4 \log 10$ increase in colony forming units per ml over 24 hours). Keeping sponges hanging in a moist shower may have allowed $\mathbf{P}$ aeruginosa to proliferate.

Because this organism has been recovered from sinks, baths, and tap water, inadequately maintained hot tubs, spas and sponges kept in such a moist environment may have contributed to this incident.

FROM: Bottone EJ, Perez II AA. J Clin Microbiol 1993;31:480-483.

\section{New AIDS Research Institute Opens in San Francisco}

The Gladstone Institute of Virology and Immunology at San Francisco General Hospital, affiliated with the University of California, San Francisco (UCSF), opened its doors in April 1993. Warner C. Greene, $\mathrm{MD}, \mathrm{PhD}$, has been named as Director. In addition to the National Institutes of Health, the Gladstone Institute is the largest AIDS research unit in the United States. With funding of \$28 million for operating expenses donated by the J. David Gladstone Institute and \$4 million from UCSF, five principal research groups will work cooperatively to study the HIV life cycle and new drugs, vaccines and treatments for HIV infection.

This is one of the few privately funded research centers in the country and has resulted from a unique partnership between the private sector, UCSF, the City of San Francisco, and the State of California.

\section{Computer Reminders D ouble Rates of Influenza Vaccination in High- Risk Patients}

Doctors who receive computer-generated reminders are twice as likely to administer influenza vaccine to patients at high risk for pulmonary disease during the winter. Dr. Clement J. McDonald and colleagues at Indiana University School of Medicine found that 